\title{
The Exp-Function Method for Solving Two Dimensional Sine-Bratu Type Equations
}

\author{
Eslam Moradi' ${ }^{1}$ Hamidreza Varasteh ${ }^{2}$, Abolfazl Abdollahzadeh ${ }^{3}$, \\ Mojtaba Mostafaei-Malekshah' 1 \\ ${ }^{1}$ Faculty of Mathematical Sciences and Computer, Kharazmi University, Tehran, Iran \\ ${ }^{2}$ Department of Mathematics, Payamenoor University, Tehran, Iran \\ ${ }^{3}$ Department of Mathematics, Faculty of Mathematical Sciences and Statistics, Birjand University, Birjand, Iran \\ Email: eslam.moradi@gmail.com, varastehhamid@gmail.com, aboalfazl.zadeh@yahoo.com, \\ m.mostafaei.m@gmail.com
}

Received 15 January 2014; revised 25 February 2014; accepted 4 March 2014

Copyright (C) 2014 by authors and Scientific Research Publishing Inc.

This work is licensed under the Creative Commons Attribution International License (CC BY). http://creativecommons.org/licenses/by/4.0/

(c) (i) Open Access

\section{Abstract \\ In this paper, we apply Exp-function method to give traveling wave solutions of second order sine- Bratu type equations. This method is straightforward, concise and effective.}

\section{Keywords}

\section{Exp-Function Method, Sine-Bratu Type Equation}

\section{Introduction}

Nonlinear differential equations have an important role in the study of nonlinear physical phenomena in science and technology. This paper investigates the exact solutions of nonlinear second order sine-Bratu type equations using the Exp-function method. Recently, several direct methods such as $\left(G^{\prime} / G\right)$-expansion method [1]-[3], sine-cosine method [4] [5], tanh-coth method [6] [7], He's homotopy perturbation method [8] [9], F-expansion method [10] [11] and others have been proposed to obtain exact solutions of nonlinear partial differential equations. Using these methods many exact solutions, including the solitary wave solutions, shock wave solutions and periodic wave solutions are obtained for some kinds of nonlinear evolution equations. The application of Exp-function method to obtain more explicit traveling wave solutions to many nonlinear differential equations has been developed by many researchers [12]-[15]. The Exp-function method is based on the assumption that the travelling wave solutions can be expressed by a polynomial in Exp-function. It has been shown that this method is direct, concise, basic and effective. The solution procedure of this method, by the help of Maple, Matlab, or any mathematical package, is of utter simplicity. 


\section{Exp-Function Method}

We suppose that the given nonlinear differential $u(x)$ to be in the form

$$
P\left(u, u_{x}, u_{y}, u_{x x}, u_{y y}, \cdots\right)=0 .
$$

where $P$ is a multivariate polynomial in its arguments. In the following, it is explained the basic for implementing Exp-function method. Taking the change of variable $\xi=k x+w y$, gives $u(x)=U(\xi)$, where $k$ and $w$ are constants parameter to be determined later. Substituting $\xi=k x+w y$ into the Equation (2.1) yields an ODEs for $U(\xi)$ of the form

$$
Q\left(U, k U^{\prime}, w U^{\prime}, k^{2} U^{\prime \prime}, w^{2} U^{\prime \prime}, \cdots\right)=0
$$

where $U^{(k)}=\frac{\mathrm{d}^{k}}{\mathrm{~d} \xi^{k}} U(\xi)$. So, if possible, integrate Equation (2.2), term by term one or more times. This introduces one or more constants of integration.

According to this method, we introduce the approach

$$
U(\xi)=\frac{a_{m} \exp (m \xi)+\cdots+a_{-n} \exp (-n \xi)}{b_{p} \exp (p \xi)+\cdots+b_{-q} \exp (-q \xi)}
$$

where $m, n, p$, and $q$ are positive integers, which could be freely chosen, $a_{i}$ for $i=-n, \cdots, m$ and $b_{j}$ for $j=-q, \cdots, p$ are unknown constants to be determined. Substituting Equation (2.3) into Equation (2.2) yields an algebraic equation in powers of the Exp-function. Then, to determine the values of $m$ and $p$, we balance the linear and nonlinear terms of the highest order in the resulting algebraic equation. Similarly, to determine the values of $n$ and $q$, we balance the linear terms of the lowest order in Equation (2.2) with the lowest order nonlinear terms.

\section{Application of the Exp-Function Method}

Consider the following sine-Bratu type equation

$$
\Delta u(x, y)+\lambda \sin (u(x, y))=0 .
$$

In order to apply the $\left(G^{\prime} / G\right)$ method, we use the transformation $\xi=k x+w t$, and change Equation (3.1) into the form

$$
\left(k^{2}+w^{2}\right) U^{\prime \prime}+\lambda \sin U=0 .
$$

And then we use the transformation $v(\xi)=\exp (i U(\xi))$, so that

$$
\sin U=\frac{v-v^{-1}}{2 i}, \quad \cos U=\frac{v+v^{-1}}{2},
$$

which gives

$$
U=\operatorname{Arccos}\left(\frac{v+v^{-1}}{2}\right)
$$

This transformation will change Equation (3.2) into the ODE in the form

$$
2\left(k^{2}+w^{2}\right)\left(v^{\prime \prime} v-v^{\prime 2}\right)+\lambda\left(v^{3}-v\right)=0
$$

where $v^{\prime}=\frac{\mathrm{d} v}{\mathrm{~d} \xi}, \quad v^{\prime \prime}=\frac{\mathrm{d}^{2} v}{\mathrm{~d} \xi^{2}}$. Now, we assume that the solution of Equation (3.3) can be expressed in the form shown in Equation (2.3). To determine the constants $m$ and $p$, we balance $v v^{\prime \prime}$ with $v^{3}$, by simple calculation, we have

$$
v v^{\prime \prime}=\frac{c_{1} \exp [(2 m+3 p) \xi]+\cdots}{c_{2} \exp [5 p \xi]+\cdots}
$$


and

$$
v^{3}=\frac{c_{3} \exp [3 m \xi]+\cdots}{c_{4} \exp [3 p \xi]+\cdots}=\frac{c_{3} \exp [(3 m+2 p) \xi]+\cdots}{c_{4} \exp [5 p \xi]+\cdots} .
$$

then we get $2 m+3 p=3 m+2 p$ which yields the leading term order $m=p$. Similarly, we balance the lowest order terms in Equations (3.4) and (3.5), to determine the values of $n$ and $q$, we obtain $2 n+3 q=3 n+2 q$ which leads to the result $n=q$.

It is possible to choose the difference values of $m, n, p$ and $q$. It is seen that when the equation has multiple solutions (like solitons) the Exp-function method is able to give us these solutions with the aid of using different $m, n, p$ and $q$. Although it worth mentioning that different parameters may lead to equivalent solutions.

Case 1: $p=m=1$ and $q=n=1$

For simplicity, we choose $p=m=1$ and $q=n=1$, the trial function, Equation (2.3) becomes as

$$
v(\xi)=\frac{a_{1} \exp (\xi)+a_{0}+a_{-1} \exp (-\xi)}{\exp (\xi)+b_{0}+b_{-1} \exp (-\xi)}
$$

Substituting Equation (3.6) into Equation (3.3) and taking the coefficients of $\exp (n \xi)$ in each term zero yield a set of algebraic equations for $a_{1}, a_{0}, a_{-1}, b_{0}, b_{-1}, w$, and $k$. Solving this system of algebraic equations with the aid of Maple, we obtain

$$
w=w, k^{2}=\lambda-w^{2}, a_{-1}=-\frac{1}{4} b_{0}^{2}, a_{0}=b_{0}, a_{1}=-1, b_{-1}=\frac{1}{4} b_{0}^{2}, b_{0}=b_{0},
$$

where $w$ and $b_{0}$ are free parameters. Substituting Equation (3.7) into Equation (3.6), it is obtained the following exact solution

$$
v_{1}(\xi)=\frac{-\exp (\xi)+b_{0}-\frac{1}{4} \exp (-\xi)}{\exp (\xi)+b_{0}+\frac{1}{4} \exp (-\xi)}=-1+\frac{2 b_{0}}{\exp (\xi)+b_{0}+\frac{1}{4} \exp (-\xi)},
$$

SO

$$
u_{1}(x, y)=\pi-\operatorname{Arccos}\left(1-\frac{2 b_{0}}{\left(\exp (\xi)+\frac{1}{4} \exp (-\xi)\right)^{2}-b_{0}^{2}}\right)
$$

Case 2: $p=m=2$ and $q=n=1$

When the parameters $p, m, q$ and $n$ are chosen $p=m=2$ and $q=n=1$, the solution is of the following form:

$$
v(\xi)=\frac{a_{2} \exp (2 \xi)+a_{1} \exp (\xi)+a_{0}+a_{-1} \exp (-\xi)}{\exp (2 \xi)+b_{1} \exp (\xi)+b_{0}+b_{-1} \exp (-\xi)} .
$$

Proceeding in a similar way as illustrated in case 1 , we can identify parameters, $a_{2}, a_{1}, a_{0}, a_{-1}, b_{1}, b_{0}, b_{-1}, w$, and $k$ in Equation (3.9) as follows:

$$
\begin{aligned}
& w=w, k^{2}=\lambda-w^{2}, a_{-1}=\frac{1}{32}\left(a_{1}+b_{1}\right)^{2}\left(a_{1}-b_{1}\right), \\
& a_{0}=-\frac{1}{16}\left(5 a_{1}-3 b_{1}\right)\left(a_{1}+b_{1}\right), a_{1}=a_{1}, \\
& a_{2}=-1, b_{-1}=-\frac{1}{32}\left(a_{1}+b_{1}\right)^{2}\left(a_{1}-b_{1}\right), \\
& b_{0}=-\frac{1}{16}\left(3 a_{1}-5 b_{1}\right)\left(a_{1}+b_{1}\right), b_{1}=b_{1},
\end{aligned}
$$


where $w, a_{1}$ and $b_{1}$ are free parameters. Similarly

$$
\begin{aligned}
v_{2}(\xi) & =\frac{-\exp (2 \xi)+a_{1} \exp (\xi)-\frac{1}{16}\left(5 a_{1}-3 b_{1}\right)\left(a_{1}+b_{1}\right)+\frac{1}{32}\left(a_{1}+b_{1}\right)^{2}\left(a_{1}-b_{1}\right) \exp (-\xi)}{\exp (2 \xi)+b_{1} \exp (\xi)-\frac{1}{16}\left(3 a_{1}-5 b_{1}\right)\left(a_{1}+b_{1}\right)-\frac{1}{32}\left(a_{1}+b_{1}\right)^{2}\left(a_{1}-b_{1}\right) \exp (-\xi)} \\
& =1+\frac{\left(a_{1}+b_{1}\right)\left[\exp (\xi)-\frac{1}{2}\left(a_{1}-b_{1}\right)\right]}{\exp (2 \xi)+b_{1} \exp (\xi)-\frac{1}{16}\left(3 a_{1}-5 b_{1}\right)\left(a_{1}+b_{1}\right)-\frac{1}{32}\left(a_{1}+b_{1}\right)^{2}\left(a_{1}-b_{1}\right) \exp (-\xi)}
\end{aligned}
$$

So,

$$
u_{2}(x, y)=\operatorname{Arccos}\left(\frac{v_{4}+v_{4}^{-1}}{2}\right)
$$

Case 3: $p=m=2$ and $q=n=2$

As mentioned earlier, the values of $m$ and $n$ can be freely chosen, now we set $p=m=2$ and $q=n=2$, Equation (2.3) turns to the following form:

$$
v(\xi)=\frac{a_{2} \exp (2 \xi)+a_{1} \exp (\xi)+a_{0}+a_{-1} \exp (-\xi)+a_{-2} \exp (-2 \xi)}{\exp (2 \xi)+b_{1} \exp (\xi)+b_{0}+b_{-1} \exp (-\xi)+b_{-2} \exp (-2 \xi)},
$$

By the same manipulation as illustrated earlier, the following relations is obtained

$$
\begin{aligned}
& w=w, k^{2}=\frac{1}{2} \lambda, a_{-2}=-\frac{1}{4} b_{0}^{2}, a_{-1}=0, a_{0}=b_{0}, a_{1}=0, \\
& a_{2}=-1, b_{-2}=-\frac{1}{4} b_{0}^{2}, b_{-1}=0, b_{0}=b_{0}, b_{1}=0,
\end{aligned}
$$

where $w$ and $b_{0}$ are free parameters. By substituting Equation (3.13) into Equation (3.12), the following exact solution is obtained

$$
v_{3}(\xi)=\frac{-\exp (2 \xi)+b_{0}-\frac{1}{4} b_{0}^{2} \exp (-2 \xi)}{\exp (2 \xi)+b_{0}+\frac{1}{4} b_{0}^{2} \exp (-2 \xi)}=-1+\frac{2 b_{0}}{\exp (2 \xi)+b_{0}+\frac{1}{4} b_{0}^{2} \exp (-2 \xi)},
$$

So

$$
u_{3}(x, y)=\operatorname{Arccos}\left(\frac{v_{3}+v_{3}^{-1}}{2}\right)
$$

or

$$
\left\{\begin{array}{l}
w=w, k^{2}=2 \lambda, \\
a_{-2}=\frac{5}{256} b_{1}^{4}-\frac{1}{16} b_{1}^{2} b_{0}+\frac{3}{128} a_{1}^{2} b_{1}^{2}+\frac{3}{64} a_{1} b_{1}^{3}-\frac{1}{64} a_{1}^{3} b_{1}-\frac{1}{16} a_{1}^{2} b_{0}-\frac{1}{8} a_{1} b_{0} b_{1}-\frac{3}{256} a_{1}^{4}, \\
a_{-1}=\frac{1}{8} a_{1}^{3}-\frac{1}{4} b_{1}^{2} a_{1}+\frac{1}{2} a_{1} b_{0}+\frac{1}{16} a_{1}^{2} b_{1}-\frac{3}{16} b_{1}^{3}+\frac{1}{2} b_{1} b_{0}, \\
a_{0}=-\frac{1}{2} a_{1}^{2}+\frac{1}{2} b_{1}^{2}-b_{0}, a_{1}=a_{1}, a_{2}=-1 \\
b_{0}=b_{0}, b_{1}=b_{1}, b_{-1}=\frac{1}{16} a_{1}^{3}-\frac{3}{16} b_{1}^{2} a_{1}+\frac{1}{2} a_{1} b_{0}-\frac{1}{8} b_{1}^{3}+\frac{1}{2} b_{1} b_{0}, \\
b_{-2}=-\frac{5}{256} b_{1}^{4}+\frac{1}{16} b_{1}^{2} b_{0}-\frac{3}{128} a_{1}^{2} b_{1}^{2}-\frac{3}{64} a_{1} b_{1}^{3}+\frac{1}{64} a_{1}^{3} b_{1}+\frac{1}{16} a_{1}^{2} b_{0}+\frac{1}{8} a_{1} b_{0} b_{1}+\frac{3}{256} a_{1}^{4}
\end{array}\right.
$$


where $w, a_{1}, b_{1}$ and $b_{0}$ are free parameters. Substituting Equation (3.15) into Equation (3.14), we obtain the following exact solution

$$
\begin{aligned}
v_{4}(\xi)= & {\left[-\exp (2 \xi)+a_{1} \exp (\xi)+\left(-\frac{1}{2} a_{1}^{2}+\frac{1}{2} b_{1}^{2}-b_{0}\right)\right.} \\
& +\left(\frac{1}{8} a_{1}^{3}-\frac{1}{4} b_{1}^{2} a_{1}+\frac{1}{2} a_{1} b_{0}+\frac{1}{16} a_{1}^{2} b_{1}-\frac{3}{16} b_{1}^{3}+\frac{1}{2} b_{1} b_{0}\right) \exp (-\xi) \\
& \left.+\left(\frac{5}{256} b_{1}^{4}-\frac{1}{6} b_{1}^{2} b_{0}+\frac{3}{128} a_{1}^{2} b_{1}^{2}+\frac{3}{64} a_{1} b_{1}^{3}-\frac{1}{64} a_{1}^{3} b_{1}-\frac{1}{16} a_{1}^{2} b_{0}-\frac{1}{8} a_{1} b_{0} b_{1}-\frac{3}{256} a_{1}^{4}\right) \exp (-2 \xi)\right] \\
& \div\left[\exp (2 \xi)+b_{1} \exp (\xi)+b_{0}+\left(\frac{1}{16} a_{1}^{3}-\frac{3}{16} b_{1}^{2} a_{1}+\frac{1}{2} a_{1} b_{0}-\frac{1}{8} b_{1}^{3}+\frac{1}{2} b_{1} b_{0}\right) \exp (-\xi)\right. \\
& \left.+\left(-\frac{5}{256} b_{1}^{4}+\frac{1}{16} b_{1}^{2} b_{0}-\frac{3}{128} a_{1}^{2} b_{1}^{2}-\frac{3}{64} a_{1} b_{1}^{3}+\frac{1}{64} a_{1}^{3} b_{1}+\frac{1}{16} a_{1}^{2} b_{0}+\frac{1}{8} a_{1} b_{0} b_{1}+\frac{3}{256} a_{1}^{4}\right) \exp (-2 \xi)\right] .
\end{aligned}
$$

So

$$
u_{4}(x, y)=\operatorname{Arccos}\left(\frac{v_{4}+v_{4}^{-1}}{2}\right)
$$

\section{Conclusion}

In this paper, we have obtained the exact solution of sine-Bratu type equation. We achieved the solution by applying Exp-function method. The results show that Exp-function method is a powerful tool for obtaining solitary solution. It may be concluded that the Exp-function method can be easily extended to all kinds of nonlinear equations. The advantage of this method over other methods is that we can obtain the exact solution by using a simple computer program. The computations associated in this work were performed by using Maple 15.

\section{References}

[1] Kutluay, S., Esen, A. and Tasbozan, O. (2010) The $\left(G^{\prime} / G\right)$-Expansion Method for Some Nonlinear Evolution Equations. Applied Mathematics and Computation, 217, 384-391. http://dx.doi.org/10.1016/j.amc.2010.05.073

[2] Aslan, I. and Ozis, T. (2009) On the Validity and Reliability of the $\left(G^{\prime} / G\right)$-Expansion Method by Using Higher-Order Nonlinear Equations. Applied Mathematics and Computation, 211, 531-536. http://dx.doi.org/10.1016/j.amc.2009.01.075

[3] Wang, M., Zhang, J. and Li, X. (2008) Application of the $\left(G^{\prime} / G\right)$-Expansion to Travelling Wave Solutions of the Broer-Kaup and the Approximate Long Water Wave Equations. Applied Mathematics and Computation, 206, 321-326. http://dx.doi.org/10.1016/j.amc.2008.08.045

[4] Wazwaz, A.M. (2006) The tanh and the sine-cosine Methods for a Reliable Treatment of the Modified Equal Width Equation and Its Variants. Communications in Nonlinear Science and Numerical Simulation, 11, 148-160. http://dx.doi.org/10.1016/j.cnsns.2004.07.001

[5] Yan, C.T. (1996) A Simple Transformation for Nonlinear Waves. Physics Letters A, 224, 77-84. http://dx.doi.org/10.1016/S0375-9601(96)00770-0

[6] Parkes, E.J. (2010) Observations on the tanh-coth Expansion Method for Finding Solutions to Nonlinear Evolution Equations. Applied Mathematics and Computation, 217, 1749-1754. http://dx.doi.org/10.1016/j.amc.2009.11.037

[7] Wazwaz, A.M. (2005) The tanh Method: Exact Solutions of the Sine-Gordon and Sinh-Gordon Equations. Applied Mathematics and Computation, 167, 1196-1210. http://dx.doi.org/10.1016/j.amc.2004.08.005

[8] Biazar, J. and Ghazvini, H. (2007) Exact Solutions for Non-Linear Schrödinger Equations by He’s Homotopy Perturbation Method. Physics Letters A, 366, 79-84. http://dx.doi.org/10.1016/j.physleta.2007.01.060

[9] He, J.H. (2005) Application of Homotopy Perturbation Method to Nonlinear Wave Equations. Chaos, Solitons \& Fractals, 26, 695-700. http://dx.doi.org/10.1016/j.chaos.2005.03.006

[10] Wang, M.L. and Li, X.Z. (2005) Extended F-Expansion Method and Periodic Wave Solutions for the Generalized 
Zakharov Equations. Physics Letters A, 343, 48-54. http://dx.doi.org/10.1016/j.physleta.2005.05.085

[11] Wang, M.L. and Li, X.Z. (2005) Applications of F-Expansion to Periodic Wave Solutions for a New Hamiltonian Amplitude Equation. Chaos, Solitons \& Fractals, 24, 1257-1268. http://dx.doi.org/10.1016/j.chaos.2004.09.044

[12] Bekir, A. and Boz, A. (2008) Exact Solutions for Nonlinear Evolution Equations Using Exp-Function Method. Physics Letters A, 372, 1619-1625. http://dx.doi.org/10.1016/j.physleta.2007.10.018

[13] Ebaid, A. (2007) Exact Solitary Wave Solutions for Some Nonlinear Evolution Equations via Exp-Function Method. Physics Letters A, 365, 213-219. http://dx.doi.org/10.1016/j.physleta.2007.01.009

[14] Abdou, M.A., Solimanm, A.A. and El-Basyony, S.T. (2007) New Application of Exp-Function Method for Improved Boussinesq Equation. Physics Letters A, 369, 469-475. http://dx.doi.org/10.1016/j.physleta.2007.05.039

[15] Zhu, S.D. (2007) Exp-Function Method for the Discrete mKdV Lattice. International Journal of Nonlinear Sciences and Numerical Simulation, 8, 465-469. 